\title{
CENÁRIO DAS REVISTAS GERIDAS PELOS PARTICIPANTES DO CURSO DE EDITORAÇÃO CIENTÍFICA DA ABEC ${ }^{i}$
}

\author{
Milton Shintaku \\ Instituto Brasileiro de Informação em Ciência e Tecnologia \\ milton.shintaku@gmail.com \\ Suely de Brito Clemente Soares \\ Associação Brasileira de Editores Científicos \\ suelybcs@gmail.com \\ Benedito Barraviera \\ Universidade Estadual Paulista \\ bbviera@gnosis.com.br \\ Suzana Caetano da Silva Lannes \\ Universidade de São Paulo \\ scslan@gmail.com \\ Sigmar de Mello Rode \\ Universidade Estadual Paulista \\ sigmarrode@uol.com.br \\ Rui Seabra Ferreira Jr \\ Associação Brasileira de Editores Científicos \\ rui.ead@gmail.com
}

\begin{abstract}
Resumo
O Curso de Editoração Científica (CEC), promovido pela Associação Brasileira de Editores Científicos (ABEC Brasil), procura discutir os principais temas de interesse dos editores brasileiros. Para tanto, buscou uma interlocução com seus participantes, por meio de um questionário, acerca do qual esse artigo apresenta uma análise. Um estudo misto, embasado na resposta de 236 participantes, revelou algumas das características das revistas desses editores, como a maioria ser de universidades e utilizarem o Open Journal System (OJS), entre outras informações. Assim, pode-se ter uma ideia do público-alvo, seus interesse e características
\end{abstract}

Palavras-chave

ABEC Brasil. Curso de Editoração Científica. Editores brasileiros.

\section{INTRODUÇÃO}

A Associação Brasileira de Editores Científicos (ABEC Brasil) é uma entidade sem fins lucrativos, que atua no desenvolvimento e aprimoramento dos periódicos científicos, por meio da congregação de pessoas físicas e jurídicas. O trabalho da ABEC Brasil é reconhecido pelos pares, tanto que Krzyzanowski e Ferreira (1998), avaliando os periódicos técnico-científicos, destaca a atuação da associação para a conscientização dos editores brasileiros, no que tange a qualidade das revistas.

Como forma de atuação, a ABEC Brasil promove a comunicação e divulgação de informações relacionadas ao processo de editoração científica, com eventos semestrais, que apoiam o amplo debate entre os participantes, mediados por especialistas. Stumpf (1998), avaliando alguns desses eventos, relata que estes refletem a preocupação da associação com a qualidade das revistas em todas as etapas da publicação. 
Um dos eventos promovidos pela ABEC Brasil é o Curso de Editoração Científica (CEC), que em 2017 teve a sua vigésima quinta edição. Dessa forma, o CEC torna-se um evento tradicional no calendário anual dos editores, não apenas pela sua continuidade, mas pela evolução dos temas tratados em cada encontro, apoiando os novos editores e a reciclagem de conhecimento para os editores mais experientes.

Com isso, a ABEC Brasil, com mais de 30 anos de atuação, tem sido um pilar na criação e aperfeiçoamento dos periódicos técnico-científicos no Brasil, com papel importante, destacando no cenário científico atual. Entretanto, para cumprir o seu papel, a associação necessita de interlocução contínua com os participantes de seus eventos, de forma a atender os anseios e verificar tendências.

Assim, o objetivo do presente estudo é apresentar os resultados da interação da ABEC Brasil com os participantes do XXV CEC, efetuados por meio de levantamento pós-evento. Com isso, procura-se conhecer mais sobre as revistas que são geridas pelos participantes de eventos da associação, de forma a planejar atividades que atendam melhor o público-alvo do evento.

\section{PERIÓDICOS CIENTÍFICOS BRASILEIROS}

Desde a sua criação, ainda no século XVII com o Journal des Savants e Philosophical Transactions of Royal Society, o periódico científico se transformou no principal canal para a disseminação de resultados de pesquisa. Muito dessa preferência se refere ao artigo, que possui o tamanho e formato apropriado para disseminar informações.

No Brasil, o periódico científico e o ensino superior possuem marcos comum, na vinda da família real fugindo de Napoleão Bonaparte. Para o ensino superior, tem-se como marco a criação do Curso Médico de Cirurgia na Bahia e Escola Anatômica,
Cirúrgica e Médica no Rio de janeiro em 1808, que foram marcos na educação brasileira (FÁVERO, 2006, p. 20). Para as publicações tem-se a criação da Imprensa Régia. As universidades só aparecerem no Brasil no início do século XIX com a Universidade Federal do Amazonas (UFAM) em 1909, muito depois da primeira universidade da América do Sul, Universidad Mayor de San Marcos, no Peru, fundada em 1551.

Os periódicos Gazeta Médica do Rio de Janeiro (1862) e Gazeta Médica da Bahia (1866) são considerados as primeiras publicações científicas no país, mesmo que tenham características de divulgação científica. Assim, somente em 1929 foi criada a Revista da Sociedade Brasileira de Ciências, atualmente chamada de Anais da Academia Brasileira de Ciências, que possui especificidades de periódico científico. Freitas (2006) afirma que periódicos científicos requerem alinhamento e suporte de instituições, o que aconteceu apenas no século XIX, explicando a criação tardia desses canais de disseminação da informação científica.

É inegável que a criação das universidades brasileiras foi decisiva para $\mathrm{O}$ desenvolvimento da ciência nacional e dos periódicos científicos. Por esse motivo, apenas a partir do início do século XIX temos efetivamente universidades de destaque, como a Universidade de São Paulo (USP) criada em 1911, e a Universidade Federal do Paraná (UFPR) em 1912 além da UFAM, mencionada anteriormente.

A criação da ABEC Brasil em 1985 revela o amadurecimento das revistas e de seus editores, da mesma forma a preocupação de criar uma associação que agregasse e disseminasse saberes sobre a editoração científica. Esse acontecimento reflete o desejo dos editores brasileiros no fomento à criação de novas revistas e melhoria de qualidade nas revistas existentes.

O advento do Scientific Eletronic Library Online (SciELO) em 1998 foi um marco na publicação científica brasileira, apresentando 
um novo modelo de negócio, com oferta do acesso livre ao conteúdo dos periódicos. Com isso, tem-se início do Movimento de Acesso Aberto no país, atuando na visibilidade e qualidade, na medida em que se tornou um indexador e um portal com características de publisher.

No que concerne à avaliação dos periódicos, na avaliação de área da Coordenação de Aperfeiçoamento de Pessoal de Nivel Superior (Capes), triênio 1997 a 2000, iniciou-se o desenho do Qualis periódico, que foi implementado em 2003, como relata Souza e Macedo (2012). Este se tornou um dos principais indicadores de qualidade e representatividade voltados aos periódicos.

Outro importante marco na publicação de periódicos científicos no país ocorreu em 2005, quando o Instituto Brasileiro de Informação em Ciência e Tecnologia (Ibict) traduziu o Open Journal System (OJS) como Serviço Eletrônico de Editoração de Revista (SEER), que se tornou a ferramenta mais utilizada no país, com mais de 1600 revistas. Assim, muitos periódicos migraram para o digital, considerando seu caráter mais econômicas, flexível em relação à serialização em fascículos, e a visibilidade, entre outros pontos apresentados por Mueller (1999).

O OJS permite a criação de portais de revistas, nos quais, em apenas uma instalação pode-se manter e gerenciar inúmeras revistas. Com isso, muitas instituições, principalmente as universidades têm criado portais de periódicos. Essa iniciativa, como defendem Garrido e Schwarz (2010), pode ser um indicador de qualidade da instituição, se observar indicadores de acesso e citação, como em metaeditoras.

\section{METODOLOGIA}

O estudo tem aspectos mistos, modalidade na qual se utilizam dados quantitativos para uma análise qualitativa, no que Cresswel (2007) considera uma pesquisa aninhada. Com isso, alinha-se a precisão dos dados quantitativos com a profundidade $\mathrm{da}$ análise qualitativa, utilizando abordagens diferentes para a etapa da coleta e análise.

Como técnica de coleta de dados utilizou-se o levantamento (survey), por meio de questionário eletrônico SurveyMonkey. O uso de questionário online possibilita uma maior participação, na medida em que o questionário fica disponível sempre e a distância dos participantes da pesquisa não interfere.

As perguntas do questionário podem ser divididas em dois grupos, sendo elas:

a) dados demográficos: descritivos, com campos abertos e de seleção, com informações gerais da revista, como nome, nome da instituição vinculada, software utilizado e outros;

b) dados de procedimentos: descritivos com campos de seleção de respostas, a fim de conhecer como as revistas operam, com informações voltadas às formas de operacionalização dos processos de publicação.

Os dados coletados são tabulados em planilhas para análise, possibilitando a geração de vários subconjuntos, a fim de possibilitar encontrar padrões. Estes dados são extraídos diretamente do SurveyMonkey para ambiente local, visto que as pesquisas feitas nesse site possuem tempo limitado, sendo retiradas após alguns dias.

\section{RESULTADOS}

A pesquisa teve 236 participações entre os dias 23 de agosto a 04 de outubro de 2017, com a colaboração significativa dos participantes, considerando que o evento contou com pouco mais de 300 participantes. Essa grande participação pode indicar a disposição dos participantes do evento no diálogo com a associação, podendo ser uma iniciativa profícua para novos estudos, que 
requeiram a cooperação dos participantes, pois a pesquisa foi totalmente opcional.

Um ponto de destaque foi que $72,5 \%$ dos respondentes são membros da associação. Entretanto, a participação de $27,5 \%$ que não são membros revela que os eventos da ABEC Brasil transcendem o círculo de associados. Com isso, pode-se verificar a importância desses eventos, que atraem editores de revistas não associadas à ABEC Brasil, mesmo que isso tenha implicações financeiras, na medida em que não associados pagam mais pela inscrição.

Com mais de um quarto dos participantes não sendo associados da $\mathrm{ABEC}$ Brasil, os dados coletados no XXV CEC revelou oportunidade de crescimento para a associação, num momento em que há a possibilidade de expansão, visto que na última mudança de estatuto, datada de 2017, a ABEC Brasil tornou-se uma associação de abrangência internacional. Este ponto também pode alertar para os desafios futuros para coordenação dos eventos da associação, na medida em que atraem não associados, cujo número pode crescer, além da possibilidade de participarem pesquisadores do exterior.

Mesmo que no CEC não haja a submissão de trabalhos, tendo um caráter exclusivamente de palestras, pode-se discutir as motivações na participação de associados e não associados conforme as motivações apresentadas por Marchiori (2006), no qual a estudiosa relata que a participação em eventos possibilita a interação com os pares, fomento na troca de ideias, aprimoramento no trabalho, entre outros motivos científicos-profissionais. A qualidade do evento também pode ser uma motivação forte, visto a tradição da associação na promoção deste evento.

Mais da metade das revistas a qual os editores participaram do XXV CEC, quase $53 \%$ são vinculadas às universidades (figura 1), sendo a principal instituição, corroborando com a premissa de que grande parte das revistas brasileiras são de instituições de ensino e pesquisa. Grande parte das universidades possuem portais e são associadas institucionais, com vários editores, sendo o principal público dos eventos.

Figura 1. Tipo de instituição mantenedora da revista.

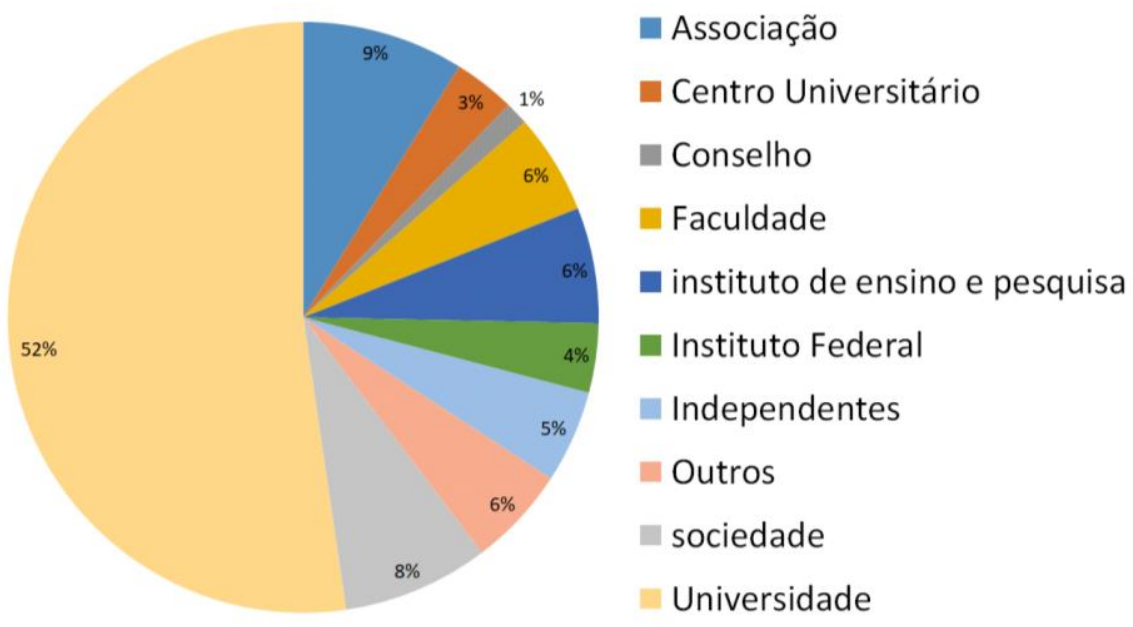

Fonte: elaborado pelos autores (2017)

A quantidade de revistas universitárias pode estar alinhada ao que Dantas (2004) defende, no qual existe a possibilidade de obter maior visibilidade das pesquisas brasileiras, com a criação de revistas científicas eletrônicas nos programas de pós-graduação. Com isso, têm-se revistas publicadas com menor custo, mesmo que dependentes de 
fomento governamental, tem uma sustentabilidade maior.

Outro ponto de destaque é a participação de editores de revistas ligadas às sociedades científicas $(9,32 \%)$ e associações científicas $(7,62 \%)$, visto que essas revistas possuem uma representatividade muito menor no cenário atual, dominado pelas revistas dos institutos de ensino e pesquisa. Entretanto, muitos desses periódicos possuem grande representatividade e historicidade, na medida em que são mais antigas que as revistas de universidades.

A presença significativa de editores de revistas de associações e sociedades científicas pode indicar o prestígio do evento, na medida em que as associações representam classes de profissionais e suas revistas são defendidas por Torres, Pardini e Ferreira (2012), em editorial para a revista Química Nova, como um bastião da independência e apropriação do conhecimento dos cientistas. Freitas (2006) relata que as associações e sociedades científicas foram às precursoras das revistas científicas no Brasil, assim como no mundo. Por isso, a participação de editores de revistas dessas entidades enriquece um evento.

A presença de revistas ligadas aos Institutos Federais (IFs), com sete editores revela uma surpresa, por serem instituições recentes. Mesmo que muitos desses institutos já existissem anteriormente, com essa nomenclatura e estatuto, são consideradas instituições recentes. Além de marcar a presença do IFs no evento, pode significar a busca da melhoria dessas instituições, na medida em que podem ministrar cursos de pós-graduação stricto sensu e revistas científicas podem ofertar mais visibilidade à instituição.

Como reflete Oranto (2010), os IFs são diferenciados por ofertarem ensino, principalmente o técnico-científico, além da pesquisa. Assim, com a criação de revistas científicas, essas instituições buscam por visibilidade na atuação em disseminação da informação científica. A participação dos editores de revistas das IFs pode indicar a preocupação na melhoria dos processos editoriais dessas revistas.

Possivelmente, o ponto de maior preocupação atual é da sustentabilidade da revista, na medida em que 182 das revistas declararam não receber fomentos externos, assumindo os custos da publicação. Das revistas que recebem subsídios, o Conselho Nacional de Desenvolvimento Científico e Tecnológico (CNPq) e a Capes foram declaradas como maiores fomentadores, apoiando 22 das revistas estudadas.

Das revistas que não recebem fomentos, cabe destacar que $16 \%$ são vinculadas às associações e sociedades científicas, que possuem orçamento próprio e o financiamento externo pode não ser um fator crucial à existência da revista. Entre as revistas das universidades que não recebem fomento (93 revistas), 27 são de instituições privadas e possuem orçamento próprio, mas 66 são de universidades públicas, que são dependentes de orçamentos cada vez mais restritos.

Uma das opções à falta de fomento é a cobrança para publicação dos artigos aceitos. Entretanto, 63,18\% ainda não utilizam essa metodologia, sendo que a instituição a qual a revista está vinculada assume as questões financeiras das revistas. Se considerarmos que a cobrança é uma tendência atual para as revistas se manterem, 30 revistas declaram cobrar por esse serviço, sendo que 21 são vinculadas às instituições públicas e 20 declararam receber fomento externo. Em relação ao valor cobrado, 15 revistas $(50 \%)$ declararam cobrar valor acima de $\mathrm{R} \$ 400,00$ (quatrocentos reais).

A cobrança por publicação tem se apresentado como uma opção ao financiamento, visto que muitas das revistas brasileiras são de acesso aberto e precisam de apoio para manter-se. Essa forma de gestão torna a revista sustentável do ponto de vista financeiro, mas acarreta alguns problemas que devem ser conduzidos, na medida em que revistas vinculadas às instituições públicas 
podem oferecer maiores dificuldades em adotar essa política.

Como relata Guanaes e Guimarães (2012), esse modelo de negócio substitui o pagamento de assinatura pela taxa de publicação. Assim, o modelo de acesso aberto aos resultados de pesquisa transfere os custos do leitor para o autor. Com isso, nos financiamentos de pesquisas, os cientistas incluem os custos de publicação nos orçamentos, de forma a possibilitar que o conhecimento circule livremente.
Em relação ao software de gestão da revista (figura 2), o OJS se apresenta como o mais utilizado, com $73 \%$ das revistas, cujos editores participaram do XXV CEC. O ScholarOne representa apenas $9,50 \%$ das revistas, muito por estar indexado pelo SciELO, que disponibiliza às revistas indexadas essa ferramenta. O restante utiliza sistemas próprios ou não utiliza sistema de publicação.

Figura 2. Ferramentas utilizadas na revista

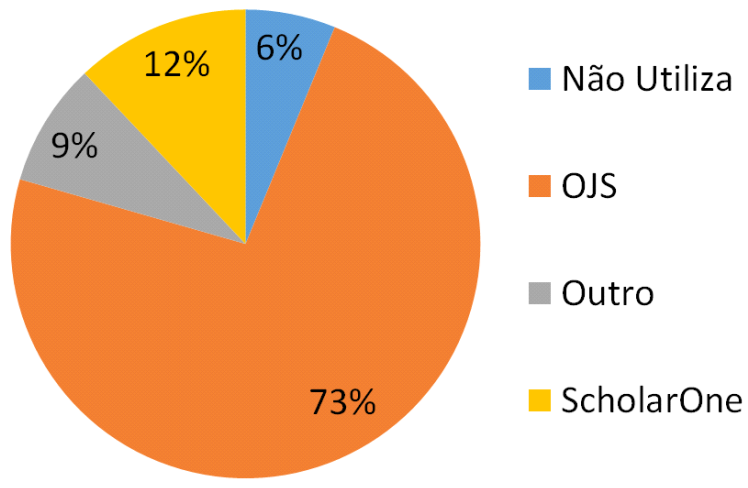

Fonte: elaborado pelos autores (2017)

Com isso, o OJS se firma como principal ferramenta utilizada no Brasil para a criação de revistas de acesso aberto, ratificando o que Baptista et al. (2007) e Shintaku, Brito e Carvalho (2014) obtiveram como resultados de estudos. Com isso, verifica-se que na amostra do estudo têm-se os mesmos resultados de pesquisas mais abrangentes, o que valida os resultados encontrados.

Em relação ao ScholarOne, Barros, Riccio e Sakata (2014) afirmam que o sistema é utilizado por vários pesquisadores em todo o mundo, o que facilita o seu uso, sendo adotado por grandes editores comerciais. Com isso, os autores afirmam que o software pode apoiar a internacionalização das revistas, na medida em que autores e pareceristas estrangeiros já estariam familiarizados com a plataforma da revista. Entretanto, por ser um sistema pago, pode apresentar desafios, principalmente porque grande parte das revistas brasileiras são de universidades públicas e que requerem financiamento para manter-se.

Independente do sistema utilizado é incontestável a necessidade de ferramenta informatizada para editoração de revista científica online, na medida em que no Brasil o papel de editor não é de tempo integral, visto que muitos professores e pesquisadores fazem essas atividades concomitantemente com outras da profissão. Nesse contexto, o desafio não está na ferramenta a ser utilizada, mas em como é possível melhorar a visibilidade da revista, considerando os desafios brasileiros em publicar ciência.

\section{CONSIDERAÇÕES FINAIS}


O cenário das revistas cujos editores participaram do XXV CEC revelou que o público-alvo do evento é predominantemente da academia, destacando-se as universidades públicas, da mesma forma que grande parte dessas revistas estão implementadas com o OJS. Assim, pode-se concluir que temas voltados a esse público-alvo podem ser mais interessantes nas próximas edições do evento, sem que se deixe de apresentar inovações e tendências mundiais, na medida em que a internacionalização dos periódicos é uma necessidade.

A cobrança parece ser uma opção para a sustentabilidade das revistas, independente se são vinculadas às instituições públicas ou privadas. Assim, pode-se tornar um tema de muito interesse para os participantes, visto que algumas revistas possuem fomento externo e cobram taxas de publicação. Esse ponto requer estudos jurídicos, na medida em que leis e orientações governamentais para instituições públicas limitam o recebimento de taxas, criando barreiras à implementação dessa política. Por isso, uma discussão mais ampla pode apoiar os editores de revistas vinculadas às instituições públicas.

Cabe ressaltar que a precisão das respostas ainda apresenta preocupações e requer processamento manual, visto diferenças de nomenclaturas utilizadas pelos respondentes. Uma grande variação de respostas pode ser verificada, revelando que análises informatizadas ainda não podem ser utilizadas em levantamentos eletrônicos. Esses problemas são mais comuns nas ciências sociais e humanas, demonstrando a riqueza e diversidade existente, assim com a complexidade, mesmo em estudos restritos.

Por fim, o presente estudo, mesmo utilizando uma amostra pequena e restrita aos participantes de um evento de uma associação, pode verificar que alguns resultados se alinham a outros estudos mais amplos. Com isso, revela a representatividade da ABEC Brasil como entidade agregadora de editores científicos brasileiros, atuando na missão de apoiar a melhoria e visibilidade da publicação científica nacional.

\title{
SCENARIO OF THE MAGAZINES MANAGED BY THE PARTICIPANTS OF ABEC'S SCIENTIFIC PUBLISHING COURSE
}

\begin{abstract}
The Scientific Publishing Course (CEC), promoted by the Braqilian Association of Scientific Editors (Abec), seeks to discuss the main topics of interest to Brazilian publishers. In order to do so, it sought a dialogue with it's participants, through a questionnaire, which this poster presents an analysis. A mixed study, based on the response of 236 participants, revealed, among others, some of the characteristics of the journals of these publishers, like most of them being from universities and using the Open Journal System (OJS). Thus, one can get an idea of the target audience, their interest and characteristics
\end{abstract}

\section{Keywords}

ABEC. Course of Scientific Publishing. Brazilian publishers.

Artigo recebido em 02/01/2018 e aceito para publicação em 17/02/2018

\section{REFERÊNCIAS}

BAPTISTA, Ana Alice et al. Comunicação científica: o papel da open archives initiative no contexto do acesso livre 10.5007/1518- 2924.2007v12nesp1p1. Encontros Bibli: revista eletrônica de biblioteconomia e ciência da informação, [s.l.], v. 12, n. 1, p.1-17, 14 dez. 2007. Universidade Federal de Santa Catarina 
Milton Shintaku, Suely de Brito Clemente Soares, Benedito Barraviera, Suzana Caetano da Silva Lannes, Sigmar de Mello Rode, Rui Seabra Ferreira Jr

(UFSC). $\quad$ http://dx.doi.org/10.5007/15182924.2007v12nesp1p1.

BARROS, Victor Freitas de Azeredo; RICCIO, Edson Luiz; SAKATA, Marici Cristine. Implementation/migration of electronic platforms of scientific journal editing: a movement towards free access to scientific information. In: $11 \mathrm{TH}$ INTERNATIONAL CONFERENCE ON INFORMATION SYSTEMS AND TECHNOLOGY MANAGEMENT CONTECSI, 11., 2014, São Paulo. Proceedings... São Paulo: Contecsi, 2014. p. 4150 - 4172. Disponível em: $<\underline{\text { http://www.contecsi.fea.usp.br/envio/11co }}$ ntecsi-

files/papers/540/submission/director/5402359-1-DR.pdf>. Acesso em: 13 dez. 2017.

CRESWELL, John W. Projeto de pesquisa: métodos qualitativo, quantitativo e misto. 3. ed. Porto Alegre: Artmed, 2010.

DANTAS, F. Responsabilidade social e pósgraduação no Brasil: ideias para (avali)ação. Revista Brasileira de Pós-Graduação, Brasília, v.1, n.2, p. 160-172, 2004. Disponível em:

$<$ http://ojs.rbpg.capes.gov.br/index.php/rbp $\mathrm{g} /$ article/viewFile/46/43>. Acesso em: 13 dez. 2017.

FÁVERO, Maria de Lourdes de Albuquerque. A universidade no Brasil: das origens à Reforma Universitária de 1968. Educar em Revista, [s.l.], n. 28, p.17-36, dez. 2006. FapUNIFESP

http://dx.doi.org/10.1590/s0104-

40602006000200003.

FÁVERO, Maria de Lourdes de Albuquerque. A universidade no Brasil: das origens à Reforma Universitária de 1968. Educar em Revista, [s.l.], n. 28, p.17-36, dez. 2006. FapUNIFESP (SciELO).
40602006000200003. Disponível em: $<\underline{\text { http://www.scielo.br/scielo.php?pid=S0104 }}$ $=$ $40602006000200003 \&$ script $=$ sci abstract $\&$ th $\mathrm{g}=\mathrm{pt}>$. Acesso em: 13 dez. 2017.

FERREIRA, Sueli Mara Soares Pinto; MARCHIORI, Patricia Zeni; CRISTOFOLI, Fulvio. Fatores motivacionais da comunidade científica para publicação e divulgação de sua produção em revistas/repositórios científicoacadêmicos: um estudo com grupos de autores-pesquisadores e Ciências da Comunicação e Ciência da Informação/Biblioteconomia. Relatório Final de Projeto. São Paulo; Curitiba: ECA-USP; DECiGI-UFPR, 2009. 61 p. Disponível em: $<$ https://core.ac.uk/download/pdf/1188580 5.pdf>. Acesso em: 13 dez. 2017.

FREITAS, Maria Helena. Considerações acerca dos primeiros periódicos científicos brasileiros. Ci. Inf., Brasília, v. 35, n. 3, p. 5466, set./dez. 2006. https://doi.org/10.18225/ci.inf..v35i3.1113. Disponível em: $<$ http://revista.ibict.br/ciinf/article/view/11 13>. Acesso em: 13 dez. 2017.

GARRIDO, Isadora dos Santos; RODRIGUES, Rosangela Schwarz. Portais de periódicos científicos online: organização institucional das publicações. Perspectivas em Ciência da Informação, Belo Horizonte, v. 15, n. 2, p. 56-72, 2010. Disponível em: $<$ http://portaldeperiodicos.eci.ufmg.br/index .php/pci/article/view/943>. Acesso em: 13 dez. 2017.

GUANAES, Paulo Cezar Vieira; GUIMARAES, Maria Cristina Soares. Acesso livre: uma nova crise no horizonte? ComCiência, Campinas, n. 139, jun. 2012. Disponível em: $<$ http://comciencia.scielo.br/scielo.php?scrip $\underline{\mathrm{t}=\text { sci } \text { arttext\&pid }=\text { S1519- }}$ 
$\underline{76542012000500008 \& \operatorname{lng}=\text { pt\&nrm }=\text { iso }}>$.

Acesso em: 02 jan. 2018.

KRZYZANOWSKI, Rosaly Favero; FERREIRA, Maria Cecília Gonzaga. Avaliação de periódicos científicos e técnicos brasileiros. Ci. Inf., Brasília, v. 27, n. 2, p. 165175 , maio/ago. 1998. https://doi.org/10.18225/ci.inf..v27i2.798. Disponível em: $<$ http://revista.ibict.br/ciinf/article/view/79 8 >. Acesso em: 13 dez. 2017.

MUELLER, Suzana P. M. O círculo vicioso que prende os periódicos nacionais. DataGramaZero: Revista de Ciência da Informação, [S.1], n. 0, dez. 1999. Disponível em:

<http://www.repositorio.unb.br/bitstream/1 0482/985/2/ARTIGO CirculoVicioso.pdf $>$. Acesso em: 13 dez. 2017.

OTRANTO, Celia Regina. Criação e implantação dos institutos federais de educação, ciência e tecnologia-IFETs. Revista Retta, Rio de Janeiro, v. 1, n.1, p. 89110, jan./jun. 2010. Disponível em: $<$ http://www.ufrri.br/SEER/index.php?jour nal $=$ retta\&page $=$ article\&op $=$ view\&path $\% 5 \mathrm{~B}$ $\% 5 \mathrm{D}=3128 \&$ path $\% 5 \mathrm{~B} \% 5 \mathrm{D}=1792>$. Acesso em: 13 dez. 2017.

i Versão revista e ampliada do Trabalho "Cenário das revistas geridas pelos participantes do curso de editoração científica da ABEC" apresentado no evento ABEC MEETING, 6-9, novembro, Curitiba, PR, 2017.
SHINTAKU, Milton; BRITO, Ronnie Fagundes de; CARVALHO NETO, Sílvio. A avaliação dos portais de revistas brasileiros implementados com o SEER/OJS por meio do levantamento da indexação pelo Latindex e SciELO. Informação \& Sociedade, João Pessoa, v. 24, n. 2, p.139-148, maio 2014. Disponível em: <http://www.ies.ufpb.br/ojs/index.php/ies/ article/view/18671>. Acesso em: $13 \mathrm{dez}$. 2017.

STUMPF, Ida Regina Chitto. Reflexões sobre as revistas brasileiras. Intexto: revista do Mestrado da Comunicação UFRGS, Porto Alegre, n. 3, p. 28-38, 1998. Disponível em: $<$ http://www.seer.ufrgs.br/index.php/intexto $\angle$ article/view/3369 > . Acesso em: $13 \mathrm{dez}$. 2017.

TORRES, Susana I. Córdoba de; PARDINI, Vera L.; FERREIRA, Vitor F. $O$ que esperamos das revistas da SBQ?. Quím. Nova, São Paulo, v. 35, n. 2, p. 233-234, 2012. http://dx.doi.org/10.1590/S010040422012000200001. Disponível em: <http://www.scielo.br/scielo.php?script=sci arttext\&pid $=\mathrm{S} 0100$ 40422012000200001\&lng $=$ en\&nrm $=$ iso $>$. Acesso em: 13 dez. 2017. 